ESAIM: M2AN 50 (2016) 995-1009

DOI: $10.1051 / \mathrm{m} 2 \mathrm{an} / 2015064$
ESAIM: Mathematical Modelling and Numerical Analysis

www.esaim-m2an.org

\title{
DOMAIN DECOMPOSITION METHOD FOR CRACK PROBLEMS WITH NONPENETRATION CONDITION
}

\author{
Evgeny RudoY ${ }^{1}$
}

\begin{abstract}
The work deals with an iteration method for numerical solving the equilibrium problem of two-dimensional elastic body with a crack under the nonpenetration condition. The method is based on the domain decomposition and Uzawa's algorithm. To construct an algorithm, the domain is partitioned into two subdomains whose common boundary contains the crack. In each subdomain the linear problems are solved. We use Lagrangian multipliers to couple the solutions and provide the nonpenetration condition on the crack.
\end{abstract}

Mathematics Subject Classification. 65N55, 65K15, 35Q74, 74R10.

Received November 17, 2014. Revised June 8, 2015.

\section{INTRODUCTION}

There are different approaches to model cracks in solids. The classical models are characterized by linear boundary conditions imposed at the crack faces [7,12,27]. It is known that such models have shortcoming because there can be situations when the crack faces penetrate each other.

It is natural to impose such boundary conditions which exclude mutual penetration of crack faces. The book [19] and parers [20-22,30,32] contain results for crack models with the non-penetration conditions for a wide class of elasticity problems. This theory is characterized by unilateral constraint conditions, and it leads to free boundary value problems.

In the present paper, the equilibrium problem of the two-dimensional elastic body with a crack is considered. The inequality type boundary conditions are imposed on the crack faces [19]. We assume a clamping condition at the part of the external boundary. The body is in equilibrium under the action of a given surface traction on the other part of the external boundary.

We use the domain decomposition method [31], based on the saddle-point theory, to construct the iteration algorithm of seeking the solution of equilibrium problem. To this end, the domain is partitioned into two subdomains in such a way that the crack is at the common boundary of subdomains. In each subdomain the linear problem of the elasticity theory is solved. Lagrangian multipliers are used for "gluing" the solutions and providing the nonpenetration conditions. The iteration algorithm is based on Uzawa's method of solution of

Keywords and phrases. Crack, nonpenetration condition, domain decomposition method, Lagrange multipliers, Uzawa's algorithm.

1 Lavrentyev Institute of Hydrodynamics SB RAS, Novosibirsk State University, 630090, Novosibirsk, Russia.

rudoi_e@mail.ru 
variational inequalities $[10,17]$. The convergence of the algorithm is proved. Numerical experiments illustrate the performance of the algorithm.

The domain decomposition method is widely used for numerical solution of many problems of mathematical physics (see, e.g., $[3,24,28,29])$. The application of the domain decomposition method to the solution of contact problems can be found in $[4,5,8,13,25]$.

There are not so many works devoted to the numerical solution of crack problems with the nonpenetration condition. In [33], a model problem for deforming an ideal elastoplastic body with a crack was investigated. For discretized problem the Uzawa algorithm was applied, but the convergence of the algorithm to the solution of continuous problem was not proved. The iterative algorithm for the solving of a crack problem based on penalty method was realized in [26]. In this case, by increase of a penalty parameter the stiffness matrix becomes illconditioned. In $[15,16]$ this approach was improved by using a primal-active set method. Numerical tests showed that the primal-active set strategy determines the exact solution of the discretized model in few iterations.

\section{Statement of the PRoblem}

Let $\Omega \subset \mathbb{R}^{2}$ be a bounded domain with Lipschitz boundary $\partial \Omega$ such that $\partial \Omega=\bar{\Gamma}_{N} \cup \bar{\Gamma}_{D}, \Gamma_{N} \cap \Gamma_{D}=\emptyset$ and meas $\Gamma_{D}>0$. Let $\Gamma_{c} \subset \Omega$ be a smooth curve without self-intersections such that $\bar{\Gamma}_{c} \cap \bar{\Gamma}_{D}=\emptyset$. Suppose that $\Omega$ is partitioned into two subdomains $\Omega_{1}$ and $\Omega_{2}$ with Lipschitz boundaries $\partial \Omega_{1}$ and $\partial \Omega_{2}$, respectively. Suppose that $\Sigma=\partial \Omega_{1} \cap \partial \Omega_{2}$. Let us consider that $\Gamma_{c} \subset \Sigma$; denote $\Gamma_{g}=\Sigma \backslash \bar{\Gamma}_{c}$. We choose the unit normal vector $\nu$ to $\Sigma$ in such a way that $\nu$ is the external normal vector to $\Omega_{1}$. Denote by $\tau$ a unit tangent vector on $\Sigma$; denote by $n$ an external normal unit vector to $\Omega$. Finally, suppose that $\Omega_{c}=\Omega \backslash \bar{\Gamma}_{c}$ is the domain with a crack.

Let $u$ be a two-component vector of displacements defined in the domain $\Omega_{c}$, i.e., $u(x): \Omega_{c} \rightarrow \mathbb{R}^{2}$; let $\sigma=\left\{\sigma_{i j}\right\}_{i, j=1}^{2}$ and $\varepsilon=\left\{\varepsilon_{i j}\right\}_{i, j=1}^{2}$ be the stress and the strain tensors which are related by linear Hooke's law:

$$
\sigma_{i j}(u)=c_{i j k l} \varepsilon_{k l}(u), \quad \varepsilon_{i j}(u)=\frac{1}{2}\left(\frac{\partial u_{i}}{\partial x_{j}}+\frac{\partial u_{j}}{\partial x_{i}}\right), \quad i, j=1,2 .
$$

The coefficients $c_{i j k l}, i, j, k, l=1,2$, satisfy the following conditions:

$$
c_{i j k l}=c_{j i k l}=c_{k l i j}, \quad a^{-1} \xi_{i j} \xi_{i j} \leq c_{i j k l} \xi_{i j} \xi_{k l} \leq a \xi_{i j} \xi_{i j} \quad \forall \xi_{i j}=\xi_{j i}
$$

for some constant $a>0$. We use the Einstein summation convention: repeated indices $i, j, k, l$ are summed from 1 to 2 .

Now, we consider the following mixed boundary value problem: for given $f \in L_{2}\left(\Gamma_{N}\right)^{2}$, find $u$ satisfying

$$
\begin{gathered}
-\operatorname{div} \sigma(u)=0 \text { in } \Omega_{c}, \\
u=0 \text { on } \Gamma_{D}, \\
\sigma(u) n=f \quad \text { on } \Gamma_{N}, \\
{[u] \cdot \nu \geq 0,\left[\sigma_{\nu}(u)\right]=0, \sigma_{\nu}(u)([u] \cdot \nu)=0 \text { on } \Gamma_{c},} \\
\sigma_{\nu}(u) \leq 0, \quad \sigma_{\tau}(u)=0 \text { on } \Gamma_{c}^{+} \cup \Gamma_{c}^{-} .
\end{gathered}
$$

Here $\Gamma_{c}^{\alpha}$ is a edge of the crack $\Gamma_{c}$ belonging to the boundary of the subdomain $\Omega_{\alpha}, \alpha=1,2 ;[v]=\left.v\right|_{\Gamma_{c}^{2}}-\left.v\right|_{\Gamma_{c}^{1}}$ is a jump of the function $v$ on $\Gamma_{c},\left.v\right|_{\Gamma_{c}^{\alpha}}$ is a trace of $v$ on $\Gamma_{c}^{\alpha} ; \sigma_{\nu}(u)=(\sigma(u) \nu) \cdot \nu$ and $\sigma_{\tau}(u)=(\sigma(u) \nu) \cdot \tau$ are normal and tangent components of the surface traction on $\Gamma_{c}$, respectively. 
Equations (2.3) and boundary conditions (2.4) - (2.6) define the displacements of the body containing the crack $\Gamma_{c}$ and being in an equilibrium under applied surface traction $f$ on $\Gamma_{N}$. Conditions (2.5)-(2.6) provide the nonpenetration of crack faces $\Gamma_{c}^{1}$ and $\Gamma_{c}^{2}$ into each other.

Let us give a variational form of the problem (2.3). To do this, we define the functional space

$$
\mathcal{V}=\left\{v \in H^{1}\left(\Omega_{c}\right)^{2} \quad v=0 \text { a.e. on } \Gamma_{D}\right\} ;
$$

the set of admissible displacements

$$
K_{c}=\left\{v \in \mathcal{V} \mid[v] \cdot \nu \geq 0 \text { a.e. on } \Gamma_{c}\right\} .
$$

Next, we define the energy functional

$$
\Pi(v)=\frac{1}{2} \int_{\Omega_{c}} \sigma(v): \varepsilon(v) \mathrm{d} x-\int_{\Gamma_{N}} f \cdot v \mathrm{~d} s,
$$

where $\sigma(v): \varepsilon(v)=\sigma_{i j}(v) \varepsilon_{i j}(v)$. The boundary value problem (2.3)-(2.5) can be formulated as the following minimization problem: find a function $u \in K_{c}$ such that

$$
\Pi(u)=\inf _{v \in K_{c}} \Pi(v) .
$$

It is known (see, e.g., [19], Thm. 1.30, p. 62) that there exists a unique solution $u \in K_{c}$ of the problem (2.7), which satisfies equation (2.3) and boundary conditions (2.4)-(2.6) in a weak sense.

\section{Domain Decomposition}

In this section, by using domain decomposition, let us rewrite problem (2.7) in an equivalent form. For this purpose, we define the following functional spaces

$$
\mathcal{V}^{\alpha}=\left\{v^{\alpha} \in H^{1}\left(\Omega_{\alpha}\right)^{2} \mid v^{\alpha}=0 \text { a.e. on } \partial \Omega_{\alpha} \cap \Gamma_{D}\right\}, \quad \alpha=1,2,
$$

and a set $K_{g c} \subset \mathcal{V}^{1} \times \mathcal{V}^{2}$, where

$$
K_{g c}=\left\{\left(v^{1}, v^{2}\right) \in \mathcal{V}^{1} \times \mathcal{V}^{2} \mid\left(v^{2}-v^{1}\right) \cdot \nu=0,\left(v^{2}-v^{1}\right) \cdot \tau=0 \text { a.e. on } \Gamma_{g},\left(v^{2}-v^{1}\right) \cdot \nu \geq 0 \text { a.e. on } \Gamma_{c}\right\} .
$$

We assume that meas $\left(\partial \Omega_{\alpha} \cup \Gamma_{D}\right)>0, \alpha=1,2$. Therefore, due to Korn's inequality and (2.2) (see, e.g. [9], Thm. 3.1, p. 115), the norm in space $\mathcal{V}^{\alpha}$ can be defined as follows

$$
\left\|v^{\alpha}\right\|_{\mathcal{V}^{\alpha}}^{2}=\int_{\Omega_{\alpha}} \sigma\left(v^{\alpha}\right): \varepsilon\left(v^{\alpha}\right) \mathrm{d} x, \quad v^{\alpha} \in \mathcal{V}^{\alpha},
$$

which is equivalent to standard norm for $\mathcal{V}^{\alpha}, \alpha=1,2$.

By using the decomposition of domain $\Omega$ into $\Omega_{1}$ and $\Omega_{2}$, we represent the energy functionals $\Pi(v)$ as sum of two functional defined on subdomains $\Omega_{1}$ and $\Omega_{2}$, i.e.,

$$
\Pi(v)=\Pi_{1}\left(v^{1}\right)+\Pi_{2}\left(v^{2}\right),
$$

where

$$
\begin{gathered}
\Pi_{\alpha}\left(v^{\alpha}\right)=\frac{1}{2} \int_{\Omega_{\alpha}} \sigma\left(v^{\alpha}\right): \varepsilon\left(v^{\alpha}\right) \mathrm{d} x-\int_{\Gamma_{N} \cap \partial \Omega_{\alpha}} f \cdot v^{\alpha} \mathrm{d} s, \\
v^{\alpha}=\left.v\right|_{\Omega_{\alpha}} \in \mathcal{V}^{\alpha}, \quad \alpha=1,2 .
\end{gathered}
$$

Consider the following minimization problem: find a pair $\left(u^{1}, u^{2}\right) \in K_{g c}$ such that

$$
\Pi_{1}\left(u^{1}\right)+\Pi_{2}\left(u^{2}\right)=\inf _{\left(v^{1}, v^{2}\right) \in K_{g c}}\left(\Pi_{1}\left(v^{1}\right)+\Pi_{2}\left(v^{2}\right)\right) .
$$


Theorem 3.1. Problem (3.1) has a unique solution $\left(u^{1}, u^{2}\right) \in K_{g c}$. Moreover,

$$
u^{\alpha}=\left.u\right|_{\Omega_{\alpha}}, \quad \alpha=1,2,
$$

where $u$ is the solution of problem (2.7).

Proof. The existence and uniqueness of the solution of problem (3.1) follows from the theory of calculus of variations (see, e.g., [10], Prop. 1.2, p. 35). Next, conditions

$$
\left(v^{2}-v^{1}\right) \cdot \nu=0, \quad\left(v^{2}-v^{1}\right) \cdot \tau=0 \quad \text { a.e. on } \quad \Gamma_{g}
$$

are equivalent to condition

$$
v^{2}-v^{1}=0 \quad \text { a.e. on } \quad \Gamma_{g} .
$$

Hence, equalities (3.2) follow from the fact that inclusion $\left(v^{1}, v^{2}\right) \in K_{g c}$ holds iff the function

$$
v(x)= \begin{cases}v^{1}(x), & \text { if } x \in \Omega_{1}, \\ v^{2}(x), & \text { if } x \in \Omega_{2}\end{cases}
$$

belongs to the set $K_{c}$. The theorem is proved.

Remark 3.2. Due to Gateaux's differentiability of the functionals $\Pi_{\alpha}, \alpha=1,2$, problem (3.1) is equivalent the following variational inequality (see, e.g., [10], Proposition 2.1, p. 38):

$$
\begin{aligned}
\int_{\Omega_{1}} \sigma\left(u^{1}\right): \varepsilon\left(v^{1}-u^{1}\right) \mathrm{d} x+\int_{\Omega_{2}} \sigma\left(u^{2}\right): & \varepsilon\left(v^{2}-u^{2}\right) \mathrm{d} x \\
& \geq \int_{\Gamma_{N} \cap \partial \Omega_{1}} f \cdot\left(v^{1}-u^{1}\right) \mathrm{d} s+\int_{\Gamma_{N} \cap \partial \Omega_{2}} f \cdot\left(v^{2}-u^{2}\right) \mathrm{d} s \quad \forall\left(v^{1}, v^{2}\right) \in K_{g c},
\end{aligned}
$$

which, in particular, implies the identity

$$
\int_{\Omega_{1}} \sigma\left(u^{1}\right): \varepsilon\left(u^{1}\right) \mathrm{d} x+\int_{\Omega_{2}} \sigma\left(u^{2}\right): \varepsilon\left(u^{2}\right) \mathrm{d} x=\int_{\Gamma_{N} \cap \partial \Omega_{1}} f \cdot u^{1} \mathrm{~d} s+\int_{\Gamma_{N} \cap \partial \Omega_{2}} f \cdot u^{2} \mathrm{~d} s .
$$

\section{Mixed VARIATIONAL FORMULATION}

We associate the Lagrange function with problem (3.1). To this end, let us define

$$
\begin{gathered}
\Lambda^{c}=\left\{\lambda^{c} \in L_{2}\left(\Gamma_{c}\right) \mid \lambda^{c} \geq 0 \text { a.e. on } \Gamma_{c}\right\}, \\
\Lambda^{\nu}=L_{2}\left(\Gamma_{g}\right), \quad \Lambda^{\tau}=L_{2}\left(\Gamma_{g}\right), \\
\Lambda=\Lambda^{c} \times \Lambda^{\nu} \times \Lambda^{\tau} .
\end{gathered}
$$

For $\lambda=\left(\lambda^{c}, \lambda^{\nu}, \lambda^{\tau}\right) \in \Lambda$ we introduce the Lagrange function for problem (2.7) as follows

$$
\begin{aligned}
L\left(v^{1}, v^{2}, \lambda\right)= & \Pi_{1}\left(v^{1}\right)+\Pi_{2}\left(v^{2}\right) \\
& +\int_{\Gamma_{c}} \lambda^{c}\left(v^{1}-v^{2}\right) \cdot \nu \mathrm{d} s+\int_{\Gamma_{g}} \lambda^{\nu}\left(v^{1}-v^{2}\right) \cdot \nu \mathrm{d} s+\int_{\Gamma_{g}} \lambda^{\tau}\left(v^{1}-v^{2}\right) \cdot \tau \mathrm{d} s .
\end{aligned}
$$


The following equality is valid

$$
\sup _{\lambda \in \Lambda} L\left(v^{1}, v^{2}, \lambda\right)= \begin{cases}\Pi_{1}\left(v^{1}\right)+\Pi_{2}\left(v^{2}\right), & \text { if }\left(v^{1}, v^{2}\right) \in K_{g c} \\ +\infty & \text { else. }\end{cases}
$$

It follows that problem (3.1) takes the following form: find a pair $\left(u^{1}, u^{2}\right) \in \mathcal{V}^{1} \times \mathcal{V}^{2}$ such that

$$
\Pi_{1}\left(u^{1}\right)+\Pi_{2}\left(u^{2}\right)=\inf _{\left(v^{1}, v^{2}\right) \in \mathcal{V}^{1} \times \mathcal{V}^{2}} \sup _{\lambda \in \Lambda} L\left(v^{1}, v^{2}, \lambda\right)
$$

Therefore, it is reasonable to connect the problem of seeking the saddle point of the Lagrangian $L$ with problem (4.1). Unfortunately, due to fact that the trace operator from $H^{1}\left(\Omega_{\alpha}\right), \alpha=1,2$, in $L_{2}\left(\Gamma_{g}\right)$ and $L_{2}\left(\Gamma_{c}\right)$ is not surjective, the well-known theorems do not guarantee the existence of the saddle point of the Lagrangian $L$. Moreover, the solution $u$ of problem (2.7) and, respectively, functions $u^{1}$ and $u^{2}$ can have a singularities in the crack tips of order $\sqrt{r}$, where $r$ is a distance to the crack tip (see, e.g., [12,20], Sect. 4.6, p. 148). Therefore, in this case there may not exist Lagrange multipliers belonging to the space $L_{2}\left(\Gamma_{g}\right)$.

In what follows, we consider the family of problems of seeking the saddle points, which depends on parameter $p>0$ and approximates problem (3.1). To this end, we use the approach applied in [6] (Chap. 5) for investigation an elasto-plastic torsion problem.

Let $p>0$; suppose that

$$
\begin{gathered}
U_{p}^{\alpha}=\left\{v^{\alpha} \in \mathcal{V}^{\alpha} \mid\left\|v^{\alpha}\right\|_{\mathcal{V}^{\alpha}} \leq p\right\}, \quad \alpha=1,2, \\
\Lambda_{p}^{c}=\left\{\lambda^{c} \in L_{2}\left(\Gamma_{c}\right) \mid 0 \leq \lambda^{c} \leq p \text { a.e. on } \Gamma_{c}\right\}, \\
\Lambda_{p}^{\nu}=\left\{\lambda^{\nu} \in L_{2}\left(\Gamma_{g}\right) \mid-p \leq \lambda^{\nu} \leq p \text { a.e. on } \Gamma_{g}\right\}, \\
\Lambda_{p}^{\tau}=\left\{\lambda^{\tau} \in L_{2}\left(\Gamma_{g}\right) \mid-p \leq \lambda^{\tau} \leq p \text { a.e. on } \Gamma_{g}\right\}, \\
\Lambda_{p}=\Lambda_{p}^{c} \times \Lambda_{p}^{\nu} \times \Lambda_{p}^{\tau} .
\end{gathered}
$$

Consider the following family of saddle point problems depending on parameter $p$ : find functions $\left(u_{p}^{1}, u_{p}^{2}, \mu_{p}\right) \in$ $U_{p}^{1} \times U_{p}^{2} \times \Lambda_{p}$ such that

$$
L\left(u_{p}^{1}, u_{p}^{2}, \lambda\right) \leq L\left(u_{p}^{1}, u_{p}^{2}, \mu_{p}\right) \leq L\left(v^{1}, v^{2}, \mu^{p}\right) \quad \forall\left(v^{1}, v^{2}, \lambda\right) \in U_{p}^{1} \times U_{p}^{2} \times \Lambda_{p} .
$$

By virtue of the fact that the sets $U_{p}^{1}, U_{p}^{2}, \Lambda_{p}$ are convex, closed and bounded in corresponding Banach spaces and the Lagrangian $L$ is convex and lower semicontinuous with respect to $\left(v^{1}, v^{2}\right)$ and concave and upper semicontinuous with respect to $\lambda$, for all $p>0$ problem (4.2) has the solution. Moreover, the pair $\left(u_{p}^{1}, u_{p}^{2}\right)$ is uniquely defined (see, e.g., [10], Proposition 2.1, p. 171 and Lem. 1.2, p. 188). 
Inequalities (4.2) are equivalent to the following variational inequalities

$$
\begin{aligned}
& \int_{\Omega_{1}} \sigma\left(u_{p}^{1}\right): \varepsilon\left(v^{1}-u_{p}^{1}\right) \mathrm{d} x+\int_{\Omega_{2}} \sigma\left(u_{p}^{2}\right): \varepsilon\left(v^{2}-u_{p}^{2}\right) \mathrm{d} x \\
& \left.+\int_{\Gamma_{c}} \mu_{p}^{c}\left(v^{1}-v^{2}-\left(u_{p}^{1}-u_{p}^{2}\right)\right) \cdot \nu\right) \mathrm{d} s+\int_{\Gamma_{g}} \mu_{p}^{\nu}\left(v^{1}-v^{2}-\left(u_{p}^{1}-u_{p}^{2}\right)\right) \cdot \nu \mathrm{d} s \\
& +\int_{\Gamma_{g}} \mu_{p}^{\tau}\left(v^{1}-v^{2}-\left(u_{p}^{1}-u_{p}^{2}\right)\right) \cdot \tau \mathrm{d} s \\
& \geq \int_{\Gamma_{N} \cap \partial \Omega_{1}} f \cdot\left(v^{1}-u_{p}^{1}\right) \mathrm{d} s+\int_{\Gamma_{N} \cap \partial \Omega_{2}} f \cdot\left(v^{2}-u_{p}^{2}\right) \mathrm{d} s, \forall\left(v^{1}, v^{2}\right) \in U_{p}^{1} \times U_{p}^{2}, \\
& \int_{\Gamma_{c}} \lambda^{c}\left(u_{p}^{1}-u_{p}^{2}\right) \mathrm{d} s \leq \int_{\Gamma_{c}} \mu_{p}^{c}\left(u_{p}^{1}-u_{p}^{2}\right) \mathrm{d} s \quad \forall \lambda^{c} \in \Lambda_{p}^{c}, \\
& \int_{\Gamma_{g}} \lambda^{\gamma}\left(u_{p}^{1}-u_{p}^{2}\right) \cdot \gamma \mathrm{d} s \leq \int_{\Gamma_{g}} \mu_{p}^{\gamma}\left(u_{p}^{1}-u_{p}^{2}\right) \cdot \gamma \mathrm{d} s \quad \forall \lambda^{\gamma} \in \Lambda_{p}^{\gamma}, \quad \gamma=\nu, \tau .
\end{aligned}
$$

Theorem 4.1. Let $\left(u^{1}, u^{2}\right)$ be the solution of problem (3.1) and $\left(u_{p}^{1}, u_{p}^{2}, \mu_{p}\right)$ be the solution of problem (4.2); then as $p \rightarrow \infty$

$$
u_{p}^{\alpha} \rightarrow u^{\alpha} \quad \text { strongly in } \mathcal{V}^{\alpha}, \quad \alpha=1,2 .
$$

Proof. Substitution of $v^{\alpha}=0, \alpha=1,2$ into (4.3) yields

$$
\begin{aligned}
\left\|u_{p}^{1}\right\|_{\mathcal{V}^{1}}^{2}+\left\|u_{p}^{2}\right\|_{\mathcal{V}^{2}}^{2}+\int_{\Gamma_{c}} \mu_{p}^{c}\left(u_{p}^{1}-u_{p}^{2}\right) \cdot \nu \mathrm{d} s+\int_{\Gamma_{g}} & \mu_{p}^{\nu}\left(u_{p}^{1}-u_{p}^{2}\right) \cdot \nu \mathrm{d} s \\
& \quad+\int_{\Gamma_{g}} \mu_{p}^{\tau}\left(u_{p}^{1}-u_{p}^{2}\right) \cdot \tau \mathrm{d} s \leq \int_{\Gamma_{N} \cap \partial \Omega_{1}} f \cdot u_{p}^{1} \mathrm{~d} s+\int_{\Gamma_{N} \cap \partial \Omega_{2}} f \cdot u_{p}^{2} \mathrm{~d} s .
\end{aligned}
$$

Substituting $\lambda=0$ into (4.4) and (4.5), respectively, we obtain

$$
\begin{gathered}
\int_{\Gamma_{c}} \mu_{p}^{c}\left(u_{p}^{1}-u_{p}^{2}\right) \cdot \nu \mathrm{d} s \geq 0, \\
\int_{\Gamma_{g}} \mu_{p}^{\gamma}\left(u_{p}^{1}-u_{p}^{2}\right) \cdot \gamma \mathrm{d} s \geq 0, \quad \gamma=\nu, \tau .
\end{gathered}
$$

Hence, due to Hölder's inequality and the boundedness of the trace operator, from (4.7) we have

$$
\left\|u_{p}^{1}\right\|_{\mathcal{V}^{1}}^{2}+\left\|u_{p}^{2}\right\|_{\mathcal{V}^{2}}^{2} \leq \int_{\Gamma_{N} \cap \partial \Omega_{1}} f \cdot u_{p}^{1} \mathrm{~d} s+\int_{\Gamma_{N} \cap \partial \Omega_{2}} f \cdot u_{p}^{2} \mathrm{~d} s \leq K_{1}\|f\|_{L_{2}\left(\partial \Omega_{1}\right)}\left\|u_{p}^{1}\right\|_{\mathcal{V}^{1}}+K_{2}\|f\|_{L_{2}\left(\partial \Omega_{2}\right)}\left\|u_{p}^{2}\right\|_{\mathcal{V}^{2}},
$$

where $K_{\alpha}$ is the norm of the trace operator $T_{\alpha}^{\Gamma_{N}}: \mathcal{V}^{\alpha} \rightarrow H^{1 / 2}\left(\Gamma_{N}\right), \alpha=1,2$. Therefore, we get estimates

$$
\left\|u_{p}^{\alpha}\right\|_{\mathcal{V}^{\alpha}} \leq K \quad \forall p>0, \alpha=1,2
$$

where $K=\sqrt{K_{1}^{2}\|f\|_{L_{2}\left(\partial \Omega_{1}\right)}^{2}+K_{2}^{2}\|f\|_{L_{2}\left(\partial \Omega_{2}\right)}^{2}}$. 
Since sequences $\left\{u_{p}^{1}\right\}$ and $\left\{u_{p}^{2}\right\}$ are bounded, there exist subsequences $\left\{u_{\tilde{p}}^{1}\right\}$ and $\left\{u_{\tilde{p}}^{2}\right\}$ and functions $\tilde{u}^{1} \in \mathcal{V}^{1}$, $\tilde{u}^{2} \in \mathcal{V}^{2}$ such that

$$
u_{\tilde{p}}^{\alpha} \rightarrow \tilde{u}^{\alpha} \quad \text { weakly in } \mathcal{V}^{\alpha}, \alpha=1,2,
$$

as $\tilde{p} \rightarrow \infty$.

Show that $\left(\tilde{u}^{1}, \tilde{u}^{2}\right) \in K_{g c}$. To this end, first note that from inequality (4.4) it follows that

$$
\mu_{p}^{c}(x)= \begin{cases}0, & \text { if }\left(u_{p}^{1}(x)-u_{p}^{2}(x)\right) \cdot \nu(x)<0 \\ p, & \text { if }\left(u_{p}^{1}(x)-u_{p}^{2}(x)\right) \cdot \nu(x)>0 .\end{cases}
$$

Moreover, from (4.5) we get for $\gamma=\nu, \tau$

$$
\mu_{p}^{\gamma}(x)=\left\{\begin{array}{l}
-p, \quad \text { if }\left(u_{p}^{1}(x)-u_{p}^{2}(x)\right) \cdot \gamma<0 \\
p, \quad \text { if }\left(u_{p}^{1}(x)-u_{p}^{2}(x)\right) \cdot \gamma>0
\end{array}\right.
$$

Introduce notation

$$
\begin{gathered}
I_{p}^{c}=\int_{\Gamma_{c}}\left(\left(u_{p}^{1}-u_{p}^{2}\right) \cdot \nu\right)^{+} \mathrm{d} s, \\
I_{p}^{\gamma}=\int_{\Gamma_{g}}\left(\left(u_{p}^{1}-u_{p}^{2}\right) \cdot \gamma\right)^{-} \mathrm{d} s+\int_{\Gamma_{g}}\left(\left(u_{p}^{1}-u_{p}^{2}\right) \cdot \gamma\right)^{+} \mathrm{d} s, \quad \gamma=\nu, \tau,
\end{gathered}
$$

where

$$
v^{+}(x)=\left\{\begin{array}{l}
v(x), \text { if } v(x) \geq 0 \\
0, \text { if } v(x)<0
\end{array}\right.
$$

$v^{-}(x)=v^{+}(x)-v(x)$.

Note that (4.11) and (4.12) imply

$$
\begin{gathered}
\int_{\Gamma_{c}} \mu_{p}^{c}\left(u_{p}^{1}-u_{p}^{2}\right) \mathrm{d} s=p I_{p}^{c}, \\
\int_{\Gamma_{g}} \mu_{p}^{\gamma}\left(u_{p}^{1}-u_{p}^{2}\right) \cdot \gamma \mathrm{d} s=p I_{p}^{\gamma}, \quad \gamma=\nu, \tau .
\end{gathered}
$$

By using these formulas, we can rewrite (4.7) in the following form:

$$
0 \leq\left\|u_{p}^{1}\right\|_{\mathcal{V}^{1}}^{2}+\left\|u_{p}^{2}\right\|_{\mathcal{V}^{2}}^{2}+p\left(I_{p}^{c}+I_{p}^{\nu}+I_{p}^{\tau}\right) \leq \int_{\Gamma_{N} \cap \partial \Omega_{1}} f \cdot u_{p}^{1} \mathrm{~d} s+\int_{\Gamma_{N} \cap \partial \Omega_{2}} f \cdot u_{p}^{2} \mathrm{~d} s
$$

Due to (4.10), we have the following estimate

$$
0 \leq p\left(I_{p}^{c}+I_{p}^{\nu}+I_{p}^{\tau}\right) \leq M,
$$

where $M=K\|f\|_{L_{2}\left(\Gamma_{N}\right)}$. The last implies

$$
\lim _{p \rightarrow \infty}\left(I_{p}^{c}+I_{p}^{\nu}+I_{p}^{\tau}\right)=0 .
$$

We take an arbitrary nonnegative function $\phi \in C_{0}^{\infty}\left(\Gamma_{c}\right)$. We have

$$
\int_{\Gamma_{c}} \phi\left(\tilde{u}^{1}-\tilde{u}^{2}\right) \cdot \nu \mathrm{d} s=\lim _{\tilde{p} \rightarrow \infty} \int_{\Gamma_{c}} \phi\left(u_{\tilde{p}}^{1}-u_{\tilde{p}}^{2}\right) \cdot \nu \mathrm{d} s \leq\left(\max _{x \in \Gamma_{c}} \phi(x)\right) \lim _{\tilde{p} \rightarrow \infty} \int_{\Gamma_{c}}\left(\left(u_{\tilde{p}}^{1}-u_{\tilde{p}}^{2}\right) \cdot \nu\right)^{+} \mathrm{d} s=0,
$$

and, consequently, $\left(\tilde{u}^{2}-\tilde{u}^{1}\right) \cdot \nu \geq 0$ a.e. on $\Gamma_{c}$. 
Let $\psi \in C_{0}^{\infty}\left(\Gamma_{g}\right)$; then we have

$$
\int_{\Gamma_{g}} \psi\left(\tilde{u}^{1}-\tilde{u}^{2}\right) \cdot \nu \mathrm{d} s=\lim _{\tilde{p} \rightarrow \infty} \int_{\Gamma_{g}} \psi\left(u_{\tilde{p}}^{1}-u_{\tilde{p}}^{2}\right) \cdot \nu \mathrm{d} s .
$$

The following inequalities are valid:

$$
-\left(\max _{x \in \Gamma_{g}} \psi(x)\right) \int_{\Gamma_{g}}\left(\left(u_{\tilde{p}}^{1}-u_{\tilde{p}}^{2}\right) \cdot \nu\right)^{-} \mathrm{d} s \leq \int_{\Gamma_{g}} \psi\left(u_{\tilde{p}}^{1}-u_{\tilde{p}}^{2}\right) \cdot \nu \mathrm{d} s \leq\left(\max _{x \in \Gamma_{g}} \psi(x)\right) \int_{\Gamma_{g}}\left(\left(u_{\tilde{p}}^{1}-u_{\tilde{p}}^{2}\right) \cdot \nu\right)^{+} \mathrm{d} s
$$

Passing to the limit as $\tilde{p} \rightarrow \infty$, we obtain

$$
\int_{\Gamma_{c}} \phi\left(\tilde{u}^{1}-\tilde{u}^{2}\right) \cdot \nu \mathrm{d} s=0 .
$$

It means that $\left(\tilde{u}^{2}-\tilde{u}^{1}\right) \cdot \nu=0$ a.e. on $\Gamma_{g}$. Similarly, it is shown that $\left(\tilde{u}^{2}-\tilde{u}^{1}\right) \cdot \tau=0$ a.e. on $\Gamma_{g}$.

Thus, the pair $\left(\tilde{u}^{1}, \tilde{u}^{2}\right)$ belongs to the set $K_{g c}$.

Now we show that $\left(\tilde{u}^{1}, \tilde{u}^{2}\right)$ coincides with $\left(u^{1}, u^{2}\right)$. Let us take an arbitrary $\left(v^{1}, v^{2}\right) \in K_{g c}$. Then for all $\tilde{p} \geq$ $\max \left\{\left\|v^{1}\right\|_{\mathcal{V}^{1}},\left\|v^{2}\right\|_{\mathcal{V}^{2}}\right\}$ the inclusion $\left(v^{1}, v^{2}\right) \in U_{\tilde{p}}^{1} \times U_{\tilde{p}}^{2}$ is valid. By virtue of (4.8) and (4.9), from inequality $(4.3)$ for pair $\left(v^{1}, v^{2}\right) \in K_{g c} \cap\left(U_{\tilde{p}}^{1} \times U_{\tilde{p}}^{2}\right)$ we get

$$
\int_{\Omega_{1}} \sigma\left(u_{\tilde{p}}^{1}\right): \varepsilon\left(v^{1}-u_{\tilde{p}}^{1}\right) \mathrm{d} x+\int_{\Omega_{2}} \sigma\left(u_{\tilde{p}}^{2}\right): \varepsilon\left(v^{2}-u_{\tilde{p}}^{2}\right) \mathrm{d} x \geq \int_{\Gamma_{N} \cap \partial \Omega_{1}} f \cdot\left(v^{1}-u_{\tilde{p}}^{1}\right) \mathrm{d} s+\int_{\Gamma_{N} \cap \partial \Omega_{2}} f \cdot\left(v^{2}-u_{\tilde{p}}^{2}\right) \mathrm{d} s .
$$

By virtue of weak lower semicontinuity of the norm, it is possible to pass to the limit in (4.14) as $\tilde{p} \rightarrow \infty$. As a result, we have inequality

$$
\int_{\Omega_{1}} \sigma\left(\tilde{u}^{1}\right): \varepsilon\left(v^{1}-\tilde{u}^{1}\right) \mathrm{d} x+\int_{\Omega_{2}} \sigma\left(\tilde{u}^{2}\right): \varepsilon\left(v^{2}-\tilde{u}^{2}\right) \mathrm{d} x \geq \int_{\Gamma_{N} \cap \partial \Omega_{1}} f \cdot\left(v^{1}-\tilde{u}^{1}\right) \mathrm{d} s+\int_{\Gamma_{N} \cap \partial \Omega_{2}} f \cdot\left(v^{2}-\tilde{u}^{2}\right) \mathrm{d} s,
$$

that is valid for all $\left(v^{1}, v^{2}\right) \in K_{g c}$ (due to arbitrariness of the choice). Variational inequality (4.15) coincides with (3.3), which uniquely defines the pair $\left(u^{1}, u^{2}\right)$. Thus, we conclude that $\left(u_{p}^{1}, u_{p}^{2}\right)$ weakly converges to $\left(u^{1}, u^{2}\right)$ as $p \rightarrow \infty$ in $\mathcal{V}^{1} \times \mathcal{V}^{2}$.

Finally, we show that (4.6) is valid. Taking into account (4.8) and (4.9), from (4.7) we get

$$
\left\|u_{p}^{1}\right\|_{\mathcal{V}^{1}}^{2}+\left\|u_{p}^{2}\right\|_{\mathcal{V}^{2}}^{2} \leq \int_{\Gamma_{N} \cap \partial \Omega_{1}} f \cdot u_{p}^{1} \mathrm{~d} s+\int_{\Gamma_{N} \cap \partial \Omega_{2}} f \cdot u_{p}^{2} \mathrm{~d} s .
$$

By virtue of weak lower semicontinuity of the norm, after passing to the limit as $p \rightarrow \infty$ the last inequality yields

$$
\left\|u^{1}\right\|_{\mathcal{V}^{1}}^{2}+\left\|u^{2}\right\|_{\mathcal{V}^{2}}^{2} \leq \liminf _{p \rightarrow \infty}\left(\left\|u_{p}^{1}\right\|_{\mathcal{V}^{1}}^{2}+\left\|u_{p}^{2}\right\|_{\mathcal{V}^{2}}^{2}\right) \leq \limsup _{p \rightarrow \infty}\left(\left\|u_{p}^{1}\right\|_{\mathcal{V}^{1}}^{2}+\left\|u_{p}^{2}\right\|_{\mathcal{V}^{2}}^{2}\right) \leq \int_{\Gamma_{N} \cap \partial \Omega_{1}} f \cdot u^{1} \mathrm{~d} s+\int_{\Gamma_{N} \cap \partial \Omega_{2}} f \cdot u^{2} \mathrm{~d} s .
$$

It follows from (3.4) that

$$
\int_{\Gamma_{N} \cap \partial \Omega_{1}} f \cdot u^{1} \mathrm{~d} s+\int_{\Gamma_{N} \cap \partial \Omega_{2}} f \cdot u^{2} \mathrm{~d} s=\left\|u^{1}\right\|_{\mathcal{V}^{1}}^{2}+\left\|u^{2}\right\|_{\mathcal{V}^{2}}^{2} .
$$


Therefore, we get

$$
\left\|u_{p}^{1}\right\|_{\mathcal{V}^{1}}^{2}+\left\|u_{p}^{2}\right\|_{\mathcal{V}^{2}}^{2} \rightarrow\left\|u^{1}\right\|_{\mathcal{V}^{1}}^{2}+\left\|u^{2}\right\|_{\mathcal{V}^{2}}^{2}
$$

as $p \rightarrow \infty$. Since $\left(u_{p}^{1}, u_{p}^{2}\right)$ converges weakly to $\left(u_{1}, u_{2}\right)$ and the norm of $\left(u_{p}^{1}, u_{p}^{2}\right)$ converges to the norm of $\left(u_{1}, u_{2}\right)$, the $\left(u_{p}^{1}, u_{p}^{2}\right)$ converges strongly to $\left(u_{1}, u_{2}\right)$ in $\mathcal{V}^{1} \times \mathcal{V}^{2}$ as $p \rightarrow \infty$ (see, e.g., [34], Thm. 8, p. 124). The theorem is proved.

Now we show that for all "sufficiently" great $p$ the set of saddle points $\left(u_{p}^{1}, u_{p}^{2}, \mu_{p}\right)$ of the Lagrangian $L$ on the set $U_{p}^{1} \times U_{p}^{2} \times \Lambda_{p}$ coincides with the set of saddle points of the same Lagrangian on $\mathcal{V}^{1} \times \mathcal{V}^{2} \times \Lambda_{p}$. Namely, the following theorem is valid.

Theorem 4.2. For all $p>K$, where $K$ is the constant in (4.10), $\left(u_{p}^{1}, u_{p}^{2}, \mu_{p}\right)$ is the saddle point of the Lagrangian $L$ on the set $U_{p}^{1} \times U_{p}^{2} \times \Lambda_{p}$ iff it satisfies (4.4), (4.5) and the following relations:

$$
\begin{aligned}
\int_{\Omega_{\alpha}} \sigma\left(u_{p}^{\alpha}\right): \varepsilon\left(v^{\alpha}\right) \mathrm{d} x+(-1)^{\alpha+1} \int_{\Gamma_{c}} \mu_{p}^{c} v^{\alpha} \cdot \nu \mathrm{d} s+(-1)^{\alpha+1} \int_{\Gamma_{g}} \mu_{p}^{\nu} v^{\alpha} \cdot \nu \mathrm{d} s & \\
\quad+(-1)^{\alpha+1} \int_{\Gamma_{g}} \mu_{p}^{\tau} v^{\alpha} \cdot \tau \mathrm{d} s & =\int_{\Gamma_{N} \cap \partial \Omega_{\alpha}} f \cdot v^{\alpha} \mathrm{d} s \quad \forall v^{\alpha} \in \mathcal{V}^{\alpha}, \quad \alpha=1,2 .
\end{aligned}
$$

Proof. To proof the theorem, it is sufficient to show that variational inequality (4.3) is valid for all functions $\left(v^{1}, v^{2}\right) \in \mathcal{V}^{1} \times \mathcal{V}^{2}$. Indeed, in this case we can substitute $\left( \pm v^{1}+u_{p}^{1}, u_{p}^{2}\right)$ and $\left(u_{p}^{1}, \pm v^{2}+u_{p}^{2}\right)$ as test functions in order to obtain (4.16).

Let $p>K$; due to (4.10), functions $\left(u_{p}^{1}, u_{p}^{2}\right)$ belong to $U_{K}^{1} \times U_{K}^{2} \subset U_{p}^{1} \times U_{p}^{2}$. Fix the number $\delta \in(0,(p-K) / 2)$. Then the open set

$$
U\left(u_{p}^{1}, u_{p}^{2}, \delta\right)=\left\{\left(v^{1}, v^{2}\right) \in \mathcal{V}^{1} \times \mathcal{V}^{2} \mid\left\|v^{1}-u_{p}^{1}\right\|_{\mathcal{V}^{1}}<\delta,\left\|v^{2}-u_{p}^{2}\right\|_{\mathcal{V}^{2}}<\delta\right\}
$$

is contained in $U_{p}^{1} \times U_{p}^{2}$.

We take arbitrary $\left(v^{1}, v^{2}\right) \in \mathcal{V}^{1} \times \mathcal{V}^{2}$ and find $\beta>0$ such that $\beta\left\|v^{\alpha}-u_{p}^{\alpha}\right\|_{\mathcal{V}^{\alpha}}<\delta, \alpha=1$, Then the pair $\left(u_{p}^{1}+\beta\left(v^{1}-u_{p}^{1}\right), u_{p}^{2}+\beta\left(v^{2}-u_{p}^{2}\right)\right)$ belongs to the set $U\left(u_{p}^{1}, u_{p}^{2}, \delta\right) \subset U_{p}^{1} \times U_{p}^{2}$. Hence, it can be substitute into (4.3) as a test function. After calculations, we get the variational inequality (4.3) which is valid for all functions $\left(v^{1}, v^{2}\right) \in \mathcal{V}^{1} \times \mathcal{V}^{2}$. The theorem is proved.

Thus, we obtain that nonlinear problem (2.7) defined in the domain $\Omega$ is approximated by two linear problems, defined in $\Omega_{1}$ and $\Omega_{2}$ and connected by Lagrange multipliers $\mu_{p}^{c}, \mu_{p}^{\nu}$ and $\mu_{p}^{\tau}$.

\section{ITERATION PROCESS FOR APPROXIMATING PROBLEM}

The goal of this section is to construct iteration Uzawa-type algorithm for problem (4.4), (4.5) and (4.16). We consider that $p>K$, where $K$ is the constant in (4.10). By $P_{\Lambda_{p}^{c}}$ we denote the projection operator on the set $\Lambda_{p}^{c}$ in $L_{2}\left(\Gamma_{c}\right)$; by $P_{\Lambda_{p}^{\gamma}}$ we denote the projection operator on the set $\Lambda_{p}^{\gamma}$ in $L_{2}\left(\Gamma_{g}\right), \gamma=\nu, \tau$. It is easy to check that

$$
\begin{gathered}
P_{\Lambda_{p}^{c} v} v(x)= \begin{cases}0, & \text { if } v(x) \leq 0, \\
v(x), & \text { if } 0<v(x)<p, \\
p, & \text { if } v(x) \geq p,\end{cases} \\
P_{\Lambda_{p}^{\nu}} v(x)=P_{\Lambda_{p}^{\tau}} v(x)= \begin{cases}-p, & \text { if } v(x) \leq-p, \\
v(x), & \text { if }-p<v(x)<p, \\
p, & \text { if } v(x) \geq p,\end{cases}
\end{gathered}
$$


Inequalities (4.4) and (4.5) are equivalent to the fact that for any real number $\theta>0$ functions $\mu_{p}^{c} \in \Lambda_{p}^{c}$, $\mu_{p}^{\gamma} \in \Lambda_{p}^{\gamma}$ are fixed points of operators

$$
\begin{aligned}
& P_{\Lambda_{p}^{c}}\left(\lambda^{c}+\theta\left(u_{p}^{1}-u_{p}^{2}\right) \cdot \nu\right): \Lambda_{p}^{c} \rightarrow \Lambda_{p}^{c}, \\
& P_{\Lambda_{p}^{\gamma}}\left(\lambda^{\gamma}+\theta\left(u_{p}^{1}-u_{p}^{2}\right) \cdot \gamma\right): \Lambda_{p}^{\gamma} \rightarrow \Lambda_{p}^{\gamma}
\end{aligned}
$$

$\gamma=\nu, \tau$, respectively, i.e. (see [10], Example on p. 39)

$$
\begin{gathered}
\mu_{p}^{c}=P_{\Lambda_{p}^{c}}\left(\mu_{p}^{c}+\theta\left(u_{p}^{1}-u_{p}^{2}\right) \cdot \nu\right), \\
\mu_{p}^{\gamma}=P_{\Lambda_{p}^{\gamma}}\left(\mu_{p}^{\gamma}+\theta\left(u_{p}^{1}-u_{p}^{2}\right) \cdot \gamma\right), \quad \gamma=\nu, \tau .
\end{gathered}
$$

Basing on equalities (5.1) and (5.2), construct a convergent iteration algorithm to find the saddle point of Lagrangian $L$ on the set $\mathcal{V}^{1} \times \mathcal{V}^{2} \times \Lambda_{p}$.

\section{Algorithm 1.}

i. Choose $\mu^{c, 0} \in \Lambda_{p}^{c}, \mu^{\nu, 0} \in \Lambda_{p}^{\nu}$ and $\mu^{\tau, 0} \in \Lambda_{p}^{\tau}$. Set $k=0$.

ii. For each $k \geq 0$ we find $u^{1, k}$ and $u^{2, k}$ as solutions of the following variational equalities:

$$
\begin{aligned}
& \int_{\Omega_{\alpha}} \sigma\left(u^{\alpha, k}\right): \varepsilon\left(v^{\alpha}\right) \mathrm{d} x+(-1)^{\alpha+1} \int_{\Gamma_{c}} \\
& \mu^{c, k} v^{\alpha} \cdot \nu \mathrm{d} s+(-1)^{\alpha+1} \int_{\Gamma_{g}} \mu^{\nu, k} v^{\alpha} \cdot \nu \mathrm{d} s \\
& \quad+(-1)^{\alpha+1} \int_{\Gamma_{g}} \mu^{\tau, k} v^{\alpha} \cdot \tau \mathrm{d} s=\int_{\Gamma_{N} \cap \partial \Omega_{\alpha}} f \cdot v^{\alpha} \mathrm{d} s \quad \forall v^{\alpha} \in \mathcal{V}^{\alpha}, \alpha=1,2 .
\end{aligned}
$$

iii. Set

$$
\begin{gathered}
\mu^{c, k+1}=P_{\Lambda_{p}^{c}}\left(\mu^{c, k}+\theta\left(u^{1, k}-u^{2, k}\right) \cdot \nu\right), \\
\mu^{\gamma, k+1}=P_{\Lambda_{p}^{\gamma}}\left(\mu^{\gamma, k}+\theta\left(u^{1, k}-u^{2, k}\right) \cdot \gamma\right), \quad \gamma=\nu, \tau .
\end{gathered}
$$

iv. Stop or $k=k+1$, goto (ii).

Theorem 5.1. There exists $\theta^{*}$ such that for all $\theta \in\left(0, \theta^{*}\right)$ the sequences $\left\{u^{\alpha, k}\right\}$ converge to $u_{p}^{\alpha}$ strongly in $\mathcal{V}^{\alpha}$, $\alpha=1,2$.

Proof. Convergence of Algorithm 1 follows from the general theorem of Uzawa's algorithm convergence (see, e.g., [10], Prop. 1.1, p. 189 or [17], Thm. 4.49, p.118). Let us check the conditions of proposition in [10]. The sets $\Lambda_{p}$ and $U_{p}^{\alpha}, \alpha=1,2$, are non-empty closed convex sets. Moreover, $\Lambda_{p}$ is bounded. The function $\lambda \rightarrow L\left(v^{1}, v^{2}, \lambda\right)$ is affine continuous one.

Due to boundedness of linear trace operator (see, e.g., [11], Thm. 1, p. 258), the following inequality

$$
\left\|v^{\alpha}-w^{\alpha}\right\|_{L_{2}\left(\Gamma_{c}\right)} \leq G\left\|v^{\alpha}-w^{\alpha}\right\|_{\mathcal{V}^{\alpha}}, \quad \forall v^{\alpha}, w^{\alpha} \in \mathcal{V}^{\alpha},
$$

holds, where the constant $G$ depends only $\Omega_{\alpha}, \alpha=1,2$. The similar inequality takes place for $L_{2}\left(\Gamma_{g}\right)$.

Finally, by virtue of Gâteaux-differentiability of the functionals $\Pi_{\alpha}, \alpha=1,2$, and Korn's inequality, we can apply Proposition 1.1 in [10], p. 189. The theorem is proved. 


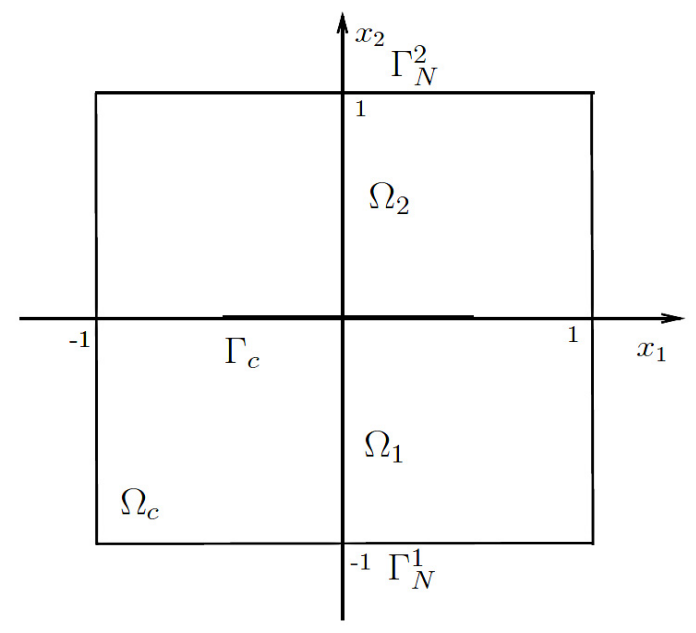

Figure 1. Domain $\Omega_{c}$ with the crack $\Gamma_{c}$.

Remark 5.2. If for each $k \geq 0$ we set $\mu^{c, k}=0$, Algorithm 1 converges to solution of the problem which approximates (as $p \rightarrow \infty$ ) the equilibrium problem of elastic body with a crack whose faces are free of traction, i.e.,

$$
\sigma_{\nu}(u)=0, \quad \sigma_{\tau}(u)=0 \quad \text { a.e. on } \quad \Gamma_{c} .
$$

\section{Numerical EXPERIMENTS}

Algorithm 1 was realized by using FreeFEM++ ([14]).

Let us consider 2D-Lamé problem with a rectilinear crack. We choose $\Omega$ as the square (see Fig. 1)

$$
\Omega=(-1,1) \times(-1,1),
$$

which decomposed into two subdomains

$$
\Omega_{1}=(-1,1) \times(-1,0), \quad \Omega_{2}=(-1,1) \times(0,1)
$$

with a common boundary

$$
\Sigma=(-1,1) \times\{0\} .
$$

Let $\Gamma_{c}=(-1 / 2,1 / 2) \times\{0\}$ be a crack; then $\Gamma_{g}=\Sigma \backslash \bar{\Gamma}_{c}=((-1,-1 / 2) \cup(1 / 2,1)) \times\{0\}$ is a part of common boundary of subdomains $\Omega_{1}$ and $\Omega_{2}$, where "gluing" occurs. The body is fixed on $\Gamma_{D}=(\{-1\} \cup\{1\}) \times(-1,1)$. By $\Gamma_{N}^{1}=(-1,1) \times\{-1\}$ and $\Gamma_{N}^{2}=(-1,1) \times\{1\}$ we denote the lower boundary and the upper boundary of square, respectively.

The plane-stress Lamé model of an isotropic solid is given in term of the stress tensor (see, e.g., [23], Example 6.5 , p. 156)

$$
\begin{gathered}
\sigma_{11}(u)=(2 \mu+\lambda) \varepsilon_{11}(u)+\lambda \varepsilon_{22}(u), \quad \sigma_{12}(u)=\sigma_{21}(u)=2 \mu \varepsilon_{12}(u), \\
\sigma_{22}(u)=\lambda \varepsilon_{11}(u)+(2 \mu+\lambda) \varepsilon_{22}(u), \\
\mu=\frac{E}{2(1+\nu)}, \quad \lambda=\frac{2 \nu \mu}{1-2 \nu},
\end{gathered}
$$

where the strain tensor is given in (2.1). We take the following values of material parameters (see [18])

$$
\nu=0.3, \quad E=6.9 \times 10^{4} \mathrm{mPa} .
$$



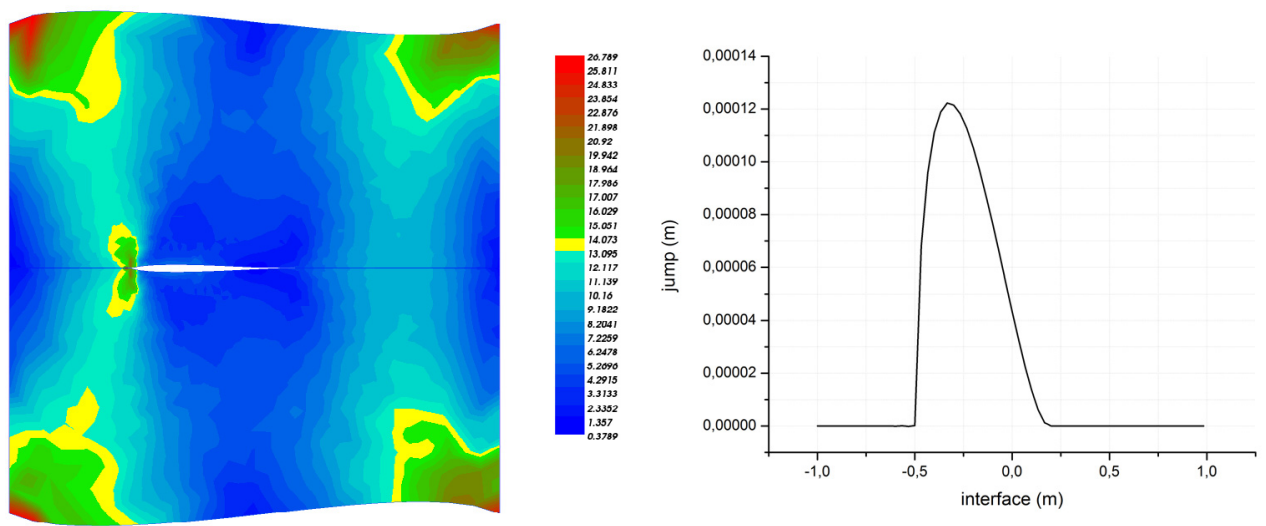

Figure 2. Partial closing of the of the crack faces.

We assume that in all numerical experiments below $\theta=2500, p=10^{7}$. The stopping criterion is

$$
\max \left(\frac{\left\|u^{1, k}-u^{1, k-1}\right\|_{\mathcal{V}^{1}}}{\left\|u^{1, k}\right\|_{\mathcal{V}^{1}}}, \frac{\left\|u^{2, k}-u^{2, k-1}\right\|_{\mathcal{V}^{2}}}{\left\|u^{2, k}\right\|_{\mathcal{V}^{2}}}\right)<10^{-6} .
$$

The spaces $\mathcal{V}_{i}, i=1,2$, are approximated by finite-element spaces consisting of piecewise linear functions Lagrange P1-elements (see, e.g., [1], Def. 6.3.5, p. 176).

Example 1 (Partial closing of the of the crack faces). At $\Gamma_{N}^{1}$ and $\Gamma_{N}^{2}$ we impose loading by the following traction forces: $f=10^{-3} \mu x$ on $\Gamma_{N}^{1}$ and $f=-10^{-3} \mu x$ on $\Gamma_{N}^{2}$. Such loading provides the closing of the crack faces in the vicinity of its right tip and opening mode in the vicinity of the left one.

Let $N$ be the number of nodes lying on the interface $\Sigma ; M$ be the number of nodes lying on the external boundary $\partial \Omega$. In this example, we shall investigate dependence of Algorithm 1 on various value $N$ and $M$. In the Table 1 we report minimal and maximal mesh sizes $h_{\min }^{\alpha}$ and $h_{\max }^{\alpha}$, the number of nodes Nodes ${ }^{\alpha}$ and triangles Triangle ${ }^{\alpha}$ in the subdomain $\Omega_{\alpha}, \alpha=1,2$; the number of iterations iter.

From the data of Table 1 we can conclude that the number of iterations practically does not depend on mesh size.

On the left in Figure 2 the domain $\Omega_{c}$ after deformation in Lagrange coordinates $x+300 u(x)$ with an amplification factor and Von Mises stresses are presented. We can see the singularities near the left tip of the crack and its absence at the right tip. This is consistent with theoretical results (see, e.g., [2]). On the right in Figure 2 the jump of normal displacements is shown.

As mentioned in the introduction, for the crack problems with linear boundary conditions imposed on cracks it is possible to get the mutual penetration of crack faces. On Figure 3 the solution of linear problem with the same loading is represented. The mesh corresponds to $N=48, M=80$; the number of iteration is 2613 . Resulting deformations and Von Mises stresses are depicted on the left; the graph of the jump of the normal displacements is given on the right. We see that the mutual penetration of crack faces occurs.

Example 2 (Opening mode). At the boundaries $\Gamma_{N}^{1}$ and $\Gamma_{N}^{2}$ we impose loading by the following traction forces: $f=-10^{-3} \mu$ on $\Gamma_{N}^{1}$ and $f=10^{-3} \mu$ on $\Gamma_{N}^{2}$. Such loading provides an opening mode of the crack.

The resulting deformations in Lagrange coordinates $x+30 u(x)$ with an amplification factor 30 and Von Mises stresses are depicted on the left in Figure 4; the jump of normal displacements along the interface $\Sigma$ is shown on the left. In this example, the number of iteration is 4508 for $N=48, M=80$. 
TABLE 1. Dependence on the mesh for the partial closing case.

\begin{tabular}{ccccccccccc}
\hline$N$ & $M$ & $h_{\min }^{1}$ & $h_{\max }^{1}$ & $h_{\min }^{2}$ & $h_{\max }^{2}$ & Nodes $^{1}$ & Triangle $^{1}$ & Nodes $^{2}$ & Triangle $^{2}$ & iter \\
\hline 12 & 32 & 0,092 & 0,365 & 0,099 & 0,365 & 111 & 180 & 116 & 190 & 2370 \\
\hline 24 & 48 & 0,041 & 0,242 & 0,044 & 0,242 & 289 & 504 & 286 & 498 & 2868 \\
48 & 80 & 0,021 & 0,157 & 0,02 & 0,157 & 921 & 1704 & 911 & 1684 & 2659 \\
96 & 144 & 0,01 & 0,086 & 0,01 & 0,086 & 3121 & 5976 & 3133 & 6000 & 2692 \\
128 & 192 & 0,0075 & 0,062 & 0,0078 & 0,062 & 5493 & 10632 & 5525 & 10696 & 2463 \\
\hline
\end{tabular}
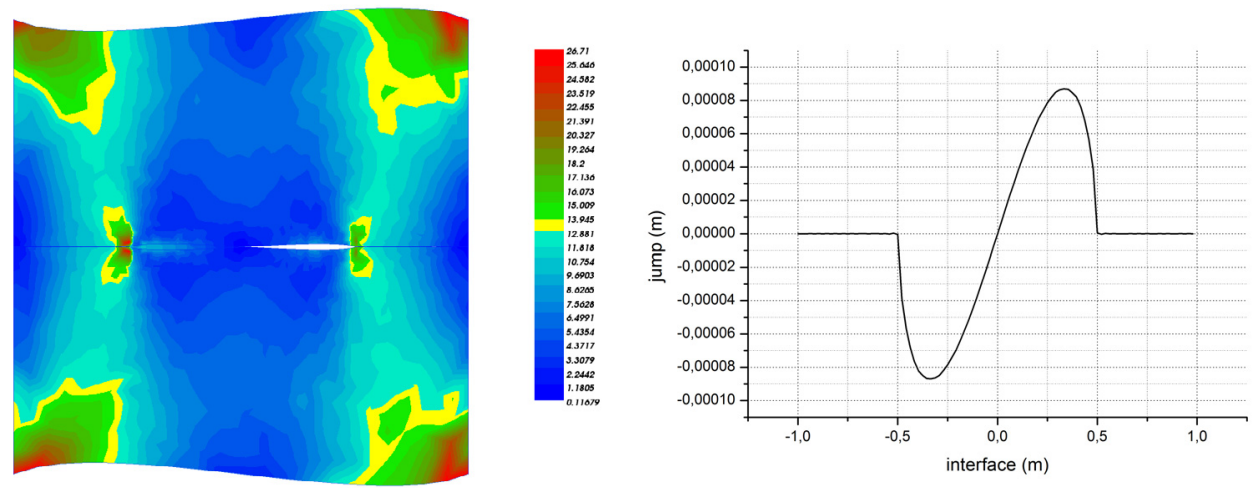

FIGURE 3. Solution of linear problem.
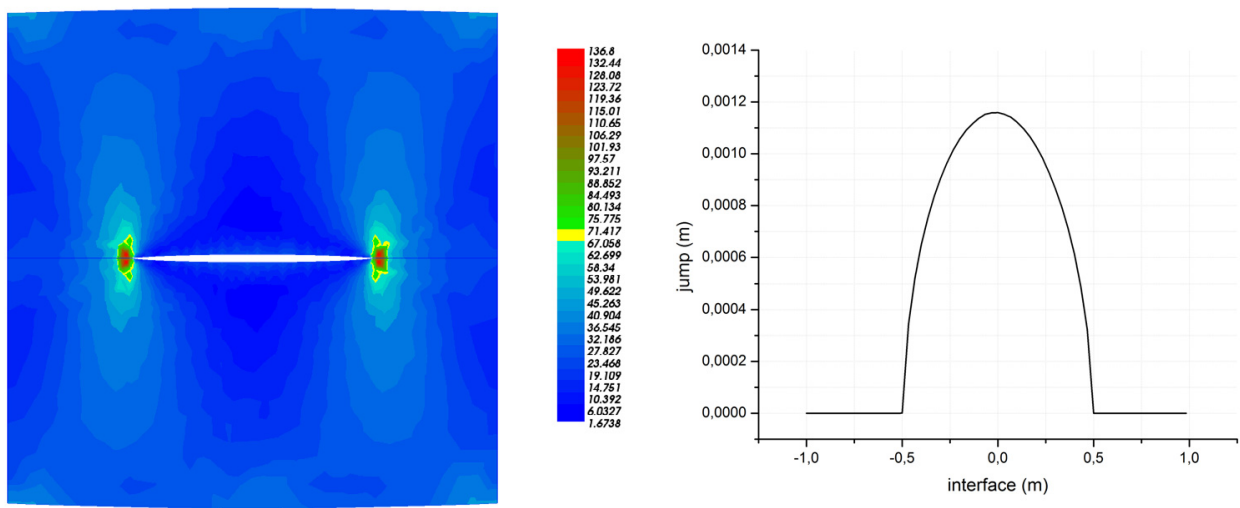

FiguRE 4. Opening mode.

Example 3 (Solution for curvilinear crack). In this example we investigate the performance of Algorithm 1 for a curvilinear crack. Let us consider the following configuration of the domain. We again choose $\Omega$ as the square with the crack

$$
\Gamma_{c}=\{(x, y) \mid y=1 / 10 \sin (2 \pi x), x \in(-1 / 2,1 / 2)\}
$$


TABLE 2. Parameters of mesh for curvilinear crack.

\begin{tabular}{cccccccccc}
\hline $\mathrm{N}$ & $\mathrm{M}$ & $h_{\min }^{1}$ & $h_{\max }^{1}$ & $h_{\min }^{2}$ & $h_{\max }^{2}$ & Nodes $^{1}$ & Triangle $^{1}$ & Nodes $^{2}$ & Triangle $^{2}$ \\
\hline 48 & 80 & 0,019 & 0,153 & 0,02 & 0,157 & 903 & 1668 & 905 & 1672 \\
\hline
\end{tabular}
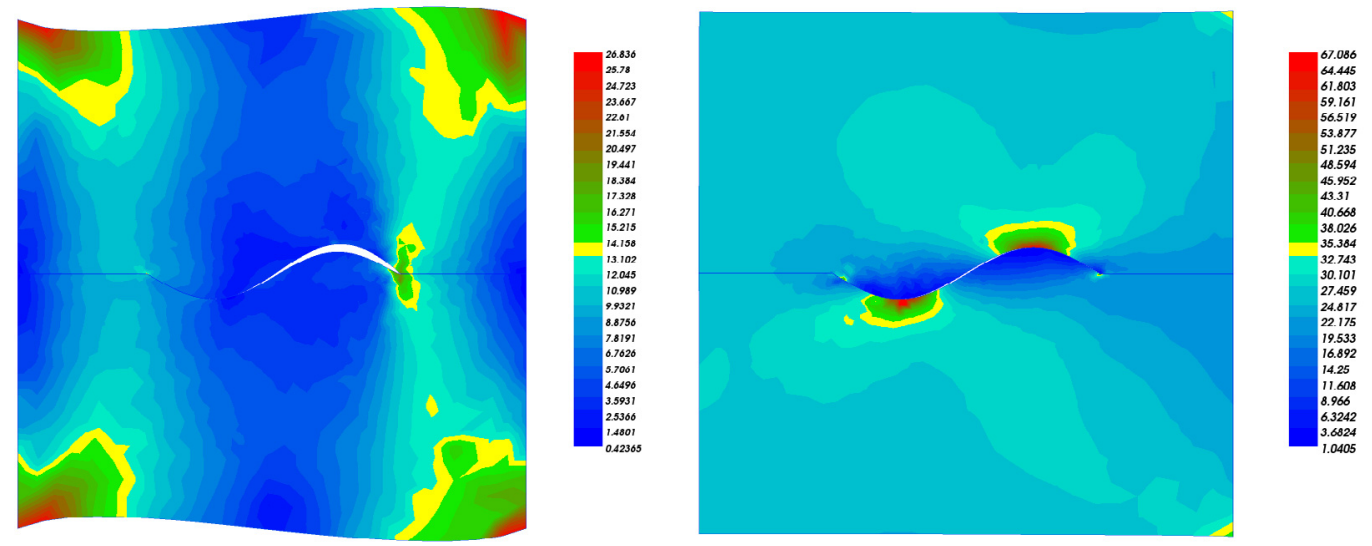

FiguRE 5. Curvilinear crack.

Let $\Gamma_{g}=\{(x, y) \quad \mid \quad y=0, x \in(-1,-1 / 2) \cup(1 / 2,1)\}$ and $\Sigma=\Gamma_{c} \cup \Gamma_{g}$. We shall consider two cases of loadings:

Case 1. We assume that the body is fix on right and left sides of the square $\Omega$. We impose loading by the following traction forces: $f=10^{-3} \mu x$ on the bottom side $(-1,1) \times\{-1\}$ and $f=-10^{-3} \mu x$ the top side $(-1,1) \times\{1\}$ of the square $\Omega$.

Case 2. We assume that the body is fix on right side of the square $\Omega$. Constant traction forces $f=10^{-3} \mu$ acts on the left sides of the square $\Omega$.

In Table 2 Parameters of mesh for both cases are given.

Resulting deformations and Von Mises stresses are depicted in Figure 5: on the left - for Case 1 (an amplification factor is equal to 300, iter $=3535$ ), on the right - for Case 2 (an amplification factor is equal to 50, iter $=4238)$.

\section{Conclusion}

The work presents the iteration algorithm of solving the equilibrium problem of two-dimensional elastic body with a crack under the nonpenetration condition. To construct the algorithm, we used the technique of domain decomposition and Uzawa's algorithm of numerical solving of problems with unilateral constraint. The suggested algorithm has advantages such as the simplicity of its realization, parallelization of computing processes, possibility of using nonconforming discretization. However, the algorithm has the disadvantage which consists in low convergence velocity. For example, the primal-dual active set strategy determines the exact solution of the discretized model in a few iterations $[15,16]$; the iterative process of penalty iteration method convergences exponentially to the solution of the penalized problem [26]. To accelerate of the Algorithm 1 we can use, for example, the augmented Lagrangian which is combination of penalty method and Lagrange multiplier approach (for notion of augmented Lagrangian see, e.g., [17]). 
Acknowledgements. This work is supported by the Grant of the President of the Russian Federation for state support of young Russian researchers (Grant No. MD-3123.2015.1).

\section{REFERENCES}

[1] G. Allaire, Numerical Analysis and Optimization: An Introduction to Mathematical Modelling and Numerical Simulation. Oxford University Press (2007).

[2] M. Bach, A.M. Khludnev and V.A. Kovtunenko, Derivatives of the energy functional for $2 D$-problems with a crack under Signorini and friction conditions. Math. Methods Appl. Sci. 23 (2000) 515-534.

[3] G.P. Astrakhantsev, Domain decomposition method for the problem of bending heterogeneous plate. Comput. Math. Math. Phys. 38 (1998) 1686-1694.

[4] G. Bayada, J. Sabil and T. Sassi, A Neumann-Neumann domain decomposition algorithm for the Signorini problem. Appl. Math. Lett. 17 (2004) 1153-1159.

[5] G. Bayada, J. Sabil and T. Sassi, Convergence of a Neumann-Dirichlet algorithm for two-body contact problems with non local Coulomb's friction law. ESAIM: M2AN 42 (2008) 243-262.

[6] J. Céa, Optimisation, Théorie et algorithmes. Dunod, Gauthier - Villars Paris (1971).

[7] G.P. Cherepanov Mechanics of Brittle Fracture. New York, McGraw-Hill (1979).

[8] J. Daněk, I. Hlaváček and J. Nedomac, Domain decomposition for generalized unilateral semi-coercive contact problem with given friction in elasticity. Math. Comput. Simul. 68 (2005) 271-300.

[9] G. Duvaut and J.-L. Lions, Inequalities in Mechanics and Physics. Springer-Verlag - Berlin, Heidelberg, New York (1976).

[10] I. Ekeland and R. Temam, Convex Analysis and Variational Problems. North-Holland Publishing Company, Amsterdam, Oxford (1976).

[11] L.C. Evans, Partial Differential Equations. AMS Press (1998).

[12] P. Grisvard, Singularities in Boundary Value Problems. Masson, Springer, Paris (1991).

[13] J. Haslinger, R. Kučera and T. Sassi, A domain decomposition algorithm for contact problems: analysis and implementation. Math. Model. Nat. Phenom. (2009) 4 123-146.

[14] F. Hecht, New development in FreeFem++. J. Numer. Math. 20 (2012) 251-265.

[15] M. Hintermüller, K. Ito and K. Kunisch, The primal-dual active set strategy as a semismooth Newton method. SIAM J. Optim. (2003) 13 865-888.

[16] M. Hintermüller, V. Kovtunenko and K. Kunisch, The primal-dual active set method for a crack problem with non-penetration. IMA J. Appl. Math. (2004) 69 1-26.

[17] K. Ito and K. Kunisch, Lagrange multiplier approach to variational problems and applications. SIAM, Philadelphia (2008).

[18] M.S.D. Jacob, P.R. Arora and M. Saleem, M.A. Elsadig and S.M. Sapuan, Fretting fatigue crack initiation: An experimental and theoretical study. Int. J. Fatigue. 29 (2007) 1328-1338.

[19] A.M. Khludnev and V.A. Kovtunenko, Analysis of cracks in solids. Southampton; WIT-Press, Boston (2000).

[20] A.M. Khludnev and V.A. Kozlov, Asymptotics of solutions near crack tips for Poisson equation with inequality type boundary conditions. Z. Angew. Math. Phys. 59 (2008) 264-280.

[21] A.M. Khludnev and G. Leugering, On elastic bodies with thin rigid inclusions and cracks. Math. Methods Appl. Sci. 33 (2010) 1955-1967.

[22] A.M. Khludnev and A. Tani, Overlapping domain problems in the crack theory with possible contact between crack faces. Q. Appl. Math. 66 (2008) 423-435.

[23] N. Kikuchi and J.T. Oden, Contact Problems in Elasticity. SIAM, Philadelphia (1988).

[24] J. Koko, Uzawa conjugate gradient domain decomposition methods for coupled Stokes flows. J. Sci. Comput. 26 (2006) $195-215$.

[25] J. Koko, Uzawa bloc relaxation domain decomposition method for a two-body frictionless contact problem. App. Math. Lett. (2009) 22 1534-1538.

[26] V.A. Kovtunenko, Numerical simulation of the non-linear crack problem with nonpenetration. Math. Meth. Appl. Sci. (2004) 27 163-179

[27] V.A. Kozlov, V.G. Mazya and J. Rossmann, Spectral Problems Associated with Corner Singularities of Solutions to Elliptic Equations. Vol. 85 of Math. Surveys and Monographs, American Mathematical Society, Providence, RI (2001).

[28] Yu.M. Laevsky, A.M. Matsokin, Decomposition methods for the solution to elliptic and parabolic boundary value problems Sib. Zh. Vychisl. Mat. 2 (1999) 361-372.

[29] E. Laitinen, A.V. Lapin and J. Pieskä, Splitting iterative methods and parallel solution of variational inequalities. Lobachevskii J. Math. 8 (2001) 167-184.

[30] N.P. Lazarev and E.M. Rudoy, Shape sensitivity analysis of Timoshenko's plate with a crack under the nonpenetration condition. Z. Angew. Math. Mech. 94 (2014) 730-739.

[31] A. Quarteroni and A. Valli, Domain decomposition methods for partial differential equations. Clarendon Press (1999).

[32] E.M. Rudoy, Shape derivative of the energy functional in a problem for a thin rigid inclusion in an elastic body. Z. Angew. Math. Phys. 66 (2015) 1923-1937.

[33] E.V. Vtorushin, Numerical investigation of a model problem for deforming an elastoplastic body with a crack under nonpenetration condition Sib. Zh. Vychisl. Mat. (2006) 9 335-344. (in Russian)

[34] K. Yosida, Functional Analysis. Springer-Verlag, Berlin Heidelberg GmbH (1968). 